\title{
INTERFERENCE OF CLOSABLE CRACKS AND NARROW SLITS IN AN ELASTIC PLATE UNDER BENDING
}

\author{
Taras M. Dalyak ${ }^{1 *}$, Ivan P. Shatskyi ${ }^{2}$ \\ ${ }^{1}$ Ivano-Frankivsk Branch of Pidstryhach-Institute for Applied Problems in Mechanics and \\ Mathematics of NAS of Ukraine, 3 Mykytynetska Street, Ivano-Frankivsk, 76002, Ukraine \\ E-mail: tdalyak@gmail.com \\ ${ }^{2}$ Ivano-Frankivsk Branch of Pidstryhach-Institute for Applied Problems in Mechanics and \\ Mathematics of NAS of Ukraine, 3 Mykytynetska Street, Ivano-Frankivsk, 76002, Ukraine \\ E-mail: ipshatsky@gmail.com \\ *corresponding author
}

\begin{abstract}
The problem of bending of an infinite plate containing an array of trough closable cracks and narrow slits is considered in a two-dimensional statement. A crack is treated as a mathematical cut, the edges of which are able to contact along the line on the plate outside. A slit is referred to as a cut with contact stress-free surfaces and the negative jump of normal displacement can occur on this cut. The crack closure caused by bending deformation was studied based on the classical hypothesis of direct normal and previously developed model of the contact of edges along the line. A new boundary problem for a couple of biharmonic equations of plane stress and plate bending with interconnected boundary conditions in the form of inequalities on the cuts is formulated. The method of singular integral equations was applied in order to develop approximate analytical and numerical solutions to the problem. The forces and moments intensity factors near the peaks of defects and contact reaction on the closed edges of the cracks are calculated. A detailed analysis was carried out for parallel rectilinear crack and slit, depending on their relative location. Presented results demonstrate qualitative differences in the stress concentration near the defects of different nature.
\end{abstract}

Keywords: Plate bending, crack closure, slit, stress intensity factor, contact reaction.

\section{Introduction}

An adequate prediction of the structural integrity of thin-walled structures is impossible without taking into account the phenomenon of the stress concentration in proximity to crack-like damages. Among the problems of the mechanics of cracks in plates and shells, there is a specific set of questions which are connected with the mutual interaction of the crack surfaces, which fall into the pressure zone. Such contact interaction of the edges of the defects can often significantly redistribute the stress field and affect the indicators of strength and durability of the construction. In the case of thin plates, these problems naturally arise when the plate is under bending strain, which gives different signs of tension on both sides of the median surface. From the mathematical standpoint, the research into the phenomenon of the closure of cracks should be conducted by 
formulating and solving problems of the elasticity theory with specific boundary conditions (inequalities) on the cuts (i.e. Khludnev and Kovtunenko 2000).

If the theory of slits or cracks with stress-free surfaces in bent plates has long been sufficiently developed (Williams (1961), Isida (1977), Berezhnitskii et al. (1979), Savruk (1981a, 1981b), Murakami (1987), Zehnder and Viz (2005)), then the new phenomenon of partial closure of through cracks by the bending of plates has only been investigated within two-dimensional theories in the last decades. The most productive model here has been that of the contact of crack edges along a line (Jones and Swedlow (1975), Heming (1980), Shatsky (1988), Young and Sun (1992), Khludnev (1995), Khludnev and Kovtunenko (2000), Shatskii (2001), Bozhidarnik et al. (2006), Lazarev (2011)). This approach was developed by Shatskii (1989), Shatsky (1998), Liu et al. (1999), Shat'skyi and Perepichka (2004), Shats'kyi and Makoviichuk (2005), Shatskyi et al. (2017) to study the behavior of closable cracks under combined tension and bending. Certain problems for arrays of rectilinear contact cracks in plates and shells have been solved in the papers by Shatskii (1990, 1991), Shatsky (1998), Perepichka (1998), Shats'kyi and Dalyak (2000), Perepichka and Shats'kyi (2002), Dalyak (2004, 2019), Opanasovych and Selivestrov (2006), Opanasovych et al. (2008), Shats'kyi and Makoviichuk (2009), Shatskii and Makoviichuk (2011), Syasky and Muzychuk (2012), Dovbnya and Shevtsova (2014), Shats'kyi (2015), Dovbnya and Hryhorchuk (2016), Opanasovych and Slobodyan (2018). In the article by Shats'kyi and Dalyak (2002), the elastic equilibrium of the bent plate containing the rectilinear cracks, connected with coaxial slits, was addressed. Shatskyi and Dalyak (2015) built an analytical solution to the problem of interaction of contact crack and collinear slot in plate bending, which in limiting case of defect blending corresponds to the results (of the above cited) source.

The purpose of this paper is to conduct analytical and numerical research of the twodimensional problem of interference of contact cracks and narrow slits in bending of the thin infinite plate. On this basis, we want to show qualitative differences in the distribution of stresses in proximity of the defects of different nature. In order to achieve the goal, we postulate the characteristic features of the two types of defects (crack and slit) and formulate the boundary problem of the classical theory of plates with conditions in the form of inequalities on the cuts contours. Hence, by the method of singular integral equations we are building an asymptotic and numerical solution to the problem and analyze the results of the calculations in case of the interaction of two parallel rectilinear cuts - a crack and a slit. Brief conclusions conclude the article. Certain data of this paper were delivered in the lecture Shatskyi and Dalyak (2018) earlier.

\section{Model and method}

\subsection{Formulation of the problem}

We shall start with the terminology and arrangements introduced by Shatskyi and Dalyak (2018). A crack is understood as a mathematical cut with zero spacing between its edges, which may contact without mutual penetration. At the same time, a slit is understood as a physical cut with small spacing between the edges. However, in a model performance we understand it as a mathematical cut with surfaces free from contact stresses, on which a negative displacement jump is possible. The level of bending load is considered to be such that the edges of the slit do not touch each other under any circumstances. This interpretation allows us to distinguish defects by type of boundary conditions.

Let us consider the infinite isotropic plate $(x, y, z) \in \mathbf{R}^{2} \times[-h, h]$ containing the array of $N$ rectilinear arbitrary oriented cuts. In the Cartesian system $x O y$, the geometry of their location is described by such parameters: $x_{n}^{0}, y_{n}^{0}$ are coordinates of the middle of $2 l_{n}$ long $n$-th cut, $\alpha_{n}$ is 
inclination angle of the defect to the abscissa axis. We dispose the local coordinate systems $x_{n} O_{n} y_{n}$ on the cuts, so that the point $O_{n}$ would be in the center of the segment $\left(-l_{n}, l_{n}\right)$, and the axis $O_{n} x_{n}$ would be direct along the defect line. Let us assume that among the $N$ cuts there are $N_{1}$ cracks, the aggregate contours of which create the set $L_{c}$. The remaining defects $\left(N-N_{1}\right)$ will be the slits with multiple contours $L_{s}$ (Fig. 1).

The plate is bent by the moments set on the cuts and on the infinity. It is necessary to study the influence of the mutual location of defects of different nature on the stressed state of the plate.

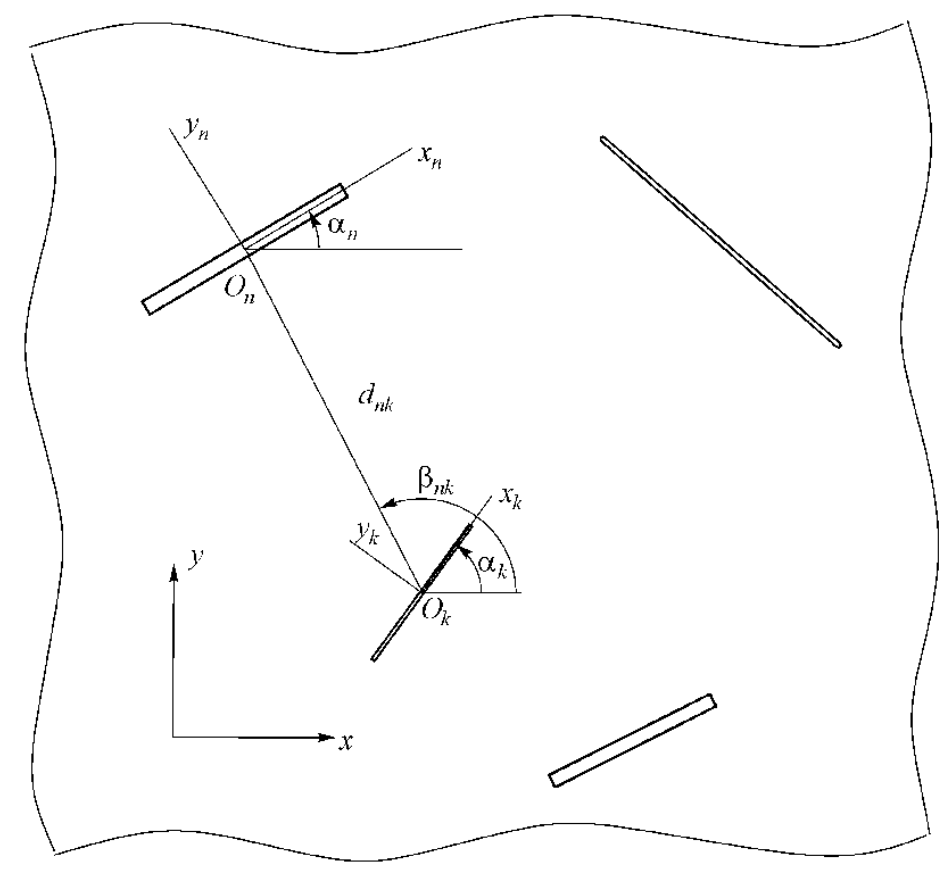

Fig. 1. The system of cracks and slits.

Consideration of the interaction of edges on at least one cut by bending will disrupt the antisymmetry of the fields of stress and displacements by thickness in the whole plate. In addition to the discontinuities of the rotation angles of the normal, jumps of the displacements appear on the cuts in the middle surface of the plate. So we describe the stress state of the plates outside of the defects using the couple of biharmonic equations corresponding to classical theories of plane stress state and plate bending:

$$
\Delta \Delta \phi=0, \quad \Delta \Delta w=0, \quad(x, y) \in \mathbf{R}^{2} \backslash\left(L_{c} \cup L_{s}\right)
$$

where $\phi$ is stress function, $w$ is plate flexure, $\Delta$ is two-dimensional Laplace operator.

The boundary conditions on the cuts depend on the type of the defects. Therefore, conditions of smooth contact of the edges along a line are set out on the edges of the cracks (Shatskyi (1988), Shatskii (1989)):

$$
\left[u_{y_{n}}\right]\left(x_{n}\right)=h\left|\left[\vartheta_{y}\right]_{n}\left(x_{n}\right)\right| \geq 0, \quad N_{x_{n} y_{n}}\left(x_{n}, 0\right)=0
$$




$$
\begin{gathered}
M_{y_{n}}\left(x_{n}, 0\right)=-m_{n}^{1}\left(x_{n}\right)+h N_{y_{n}}\left(x_{n}, 0\right) \operatorname{sgn}\left[\vartheta_{y_{n}}\right], \quad M_{x_{n} y_{n}}^{*}\left(x_{n}, 0\right)=-p_{n}^{1}\left(x_{n}\right)+C_{n} \\
N_{y_{n}}\left(x_{n}, 0\right) \leq 0, \quad x_{n} \in L_{n} \subset L_{c} ; \quad n=\overline{1, N_{1}} ;
\end{gathered}
$$

as well as conditions of the free edge for slits (Williams (1961), Isida (1977), Berezhnitskii et al. (1979), Savruk (1981a, 1981b)):

$$
\begin{gathered}
N_{y_{n}}\left(x_{n}, 0\right)=0, \quad N_{x_{n} y_{n}}\left(x_{n}, 0\right)=0 \\
M_{y_{n}}\left(x_{n}, 0\right)=-m_{n}^{1}\left(x_{n}\right), \quad M_{x_{n} y_{n}}^{*}\left(x_{n}, 0\right)=-p_{n}^{1}\left(x_{n}\right)+C_{n}, \quad x_{n} \in L_{n} \subset L_{s}, n=\overline{N_{1}+1, N}
\end{gathered}
$$

Bending and twisting moments as well as membrane forces are set up on infiniteness:

$$
M_{y}=m_{y}^{\infty}, M_{x}=m_{x}^{\infty}, M_{x y}=m_{x y}^{\infty}, N_{y}=N_{x}=N_{x y}=0,(x, y) \rightarrow \infty
$$

Here and hereafter $N_{x}, N_{y}, N_{x y}$ are membrane forces, $M_{x}, M_{y}, M_{x y}$ are moments, $M_{x y}^{*}$ is generalized torsional moment, $\left[u_{y_{n}}\right],\left[u_{x_{n}}\right]$ are the jumps of the displacements in the middle surface, $\left[\vartheta_{y_{n}}\right]=\left[\partial w / \partial y_{n}\right], \quad\left[\vartheta_{x_{n}}\right]=\left[\partial w / \partial x_{n}\right]$ are the discontinuities of the rotation angles of the normal on the cut $L_{n}, m_{n}^{1}\left(x_{n}\right), p_{n}^{1}\left(x_{n}\right)$ are of constant signs loads given on the cuts edges, $C_{n}$ are arbitrary constants.

\subsection{Integral equations}

To construct the solution to the problem (1)-(4), we used the variant of the method of singular integral equations described by Savruk (1981b). Integral expressions of the forces and moments on the cutting line $L_{n}$ irrespective of the defect type are as follows:

$$
\begin{gathered}
N_{y_{n}}\left(x_{n}, 0\right)=\frac{B}{4 \pi} \sum_{k=1}^{N} \int_{-l_{k}}^{l_{k}}\left\{K_{n k}^{11}\left(\xi, x_{n}\right)\left[u_{y_{k}}\right]^{\prime}(\xi)+K_{n k}^{12}\left(\xi, x_{n}\right)\left[u_{x_{k}}\right]^{\prime}(\xi)\right\} d \xi \\
N_{x_{n} y_{n}}\left(x_{n}, 0\right)=\frac{B}{4 \pi} \sum_{k=1}^{N} \int_{-l_{k}}^{l_{k}}\left\{K_{n k}^{21}\left(\xi, x_{n}\right)\left[u_{y_{k}}\right]^{\prime}(\xi)+K_{n k}^{22}\left(\xi, x_{n}\right)\left[u_{x_{k}}\right]^{\prime}(\xi)\right\} d \xi \\
M_{y_{n}}\left(x_{n}, 0\right)=m_{n}^{0}-\frac{D a}{4 \pi} \sum_{k=1}^{N} \int_{-l_{k}}^{l_{k}}\left\{K_{n k}^{33}\left(\xi, x_{n}\right)\left[\vartheta_{y_{k}}\right]^{\prime}(\xi)+K_{n k}^{34}\left(\xi, x_{n}\right)\left[\vartheta_{x_{k}}\right]^{\prime}(\xi)\right\} d \xi \\
M_{x_{n} y_{n}}^{*}\left(x_{n}, 0\right)=p_{n}^{0}-\frac{D a}{4 \pi} \sum_{k=1}^{N} \int_{-l_{k}}^{l_{k}}\left\{K_{n k}^{43}\left(\xi, x_{n}\right)\left[\vartheta_{y_{k}}\right]^{\prime}(\xi)+K_{n k}^{44}\left(\xi, x_{n}\right)\left[\vartheta_{x_{k}}\right]^{\prime}(\xi)\right\} d \xi
\end{gathered}
$$

Where

$m_{n}^{0}=m_{n}+m_{y}^{\infty} \cos ^{2} \alpha_{n}+m_{x}^{\infty} \sin ^{2} \alpha_{n}-m_{x y}^{\infty} \sin 2 \alpha_{n}, \quad p_{n}^{0}=m_{x y}^{\infty} \cos 2 \alpha_{n}+\left(m_{y}^{\infty}-m_{x}^{\infty}\right) \sin 2 \alpha_{n} / 2$ are functions of the so-called main stress state of the plate without defects, $B=2 E h$, $D=2 E h^{3} /\left(3\left(1-v^{2}\right)\right), a=(3+v)(1-v), E$ and $v$ are Young's modulus and Poisson's ratio for the plate material. The dash indicates the derivative with respect to a coordinate.

Kernels of expressions (5) (Savruk (1981a, 1981b)) contain singular additive of Cauchy type at $n=k$ and $i=j$, and a regular part dependent on the reciprocal location of the cuts. 
Having satisfied the boundary conditions (2) for closable cracks and (3) for narrow slits, we obtained the system of singular integral equations:

$$
\begin{aligned}
& {\left[u_{y_{n}}\right]^{\prime}\left(x_{n}\right)-h\left[\vartheta_{y n}\right]^{\prime}\left(x_{n}\right) \operatorname{sgn}\left[\vartheta_{y_{n}}\right]\left(x_{n}\right)=0} \\
& \frac{B}{4 \pi} \sum_{k=1}^{N} \int_{-l_{k}}^{l_{k}}\left\{K_{n k}^{21}\left(\xi, x_{n}\right)\left[u_{y_{k}}\right]^{\prime}(\xi)+K_{n k}^{22}\left(\xi, x_{n}\right)\left[u_{x_{k}}\right]^{\prime}(\xi)\right\} d \xi=0 \\
& \frac{B h}{4 \pi} \operatorname{sgn}\left[\vartheta_{y}\right]\left(x_{n}\right) \sum_{k=1}^{N} \int_{-l_{k}}^{l_{k}}\left\{K_{n k}^{11}\left(\xi, x_{n}\right)\left[u_{y_{k}}\right]^{\prime}(\xi)+K_{n k}^{12}\left(\xi, x_{n}\right)\left[u_{x_{k}}\right]^{\prime}(\xi)\right\} d \xi \\
& +\frac{D a}{4 \pi} \sum_{k=1}^{N} \int_{-l_{k}}^{l_{k}}\left\{K_{n k}^{33}\left(\xi, x_{n}\right)\left[\vartheta_{y_{k}}\right]^{\prime}(\xi)+K_{n k}^{34}\left(\xi, x_{n}\right)\left[\vartheta_{x_{k}}\right]^{\prime}(\xi)\right\} d \xi=m_{n}\left(x_{n}\right) \\
& \frac{D a}{4 \pi} \sum_{k=1}^{N} \int_{-l_{k}}^{l_{k}}\left\{K_{n k}^{43}\left(\xi, x_{n}\right)\left[\vartheta_{y_{k}}\right]^{\prime}(\xi)+K_{n k}^{44}\left(\xi, x_{n}\right)\left[\vartheta_{x_{k}}\right]^{\prime}(\xi)\right\} d \xi=p_{n}\left(x_{n}\right)-C_{n} \\
& x_{n} \in L_{n} \subset L_{c}, n=\overline{1, N_{1}} \\
& \frac{B}{4 \pi} \sum_{k=1}^{N} \int_{-l_{k}}^{l_{k}}\left\{K_{n k}^{11}\left(\xi, x_{n}\right)\left[u_{y_{k}}\right]^{\prime}(\xi)+K_{n k}^{12}\left(\xi, x_{n}\right)\left[u_{x_{k}}\right]^{\prime}(\xi)\right\} d \xi=0 \\
& \frac{B}{4 \pi} \sum_{k=1}^{N} \int_{-l_{k}}^{l_{k}}\left\{K_{n k}^{21}\left(\xi, x_{n}\right)\left[u_{y_{k}}\right]^{\prime}(\xi)+K_{n k}^{22}\left(\xi, x_{n}\right)\left[u_{x_{k}}\right]^{\prime}(\xi)\right\} d \xi=0 \\
& \frac{D a}{4 \pi} \sum_{k=1}^{N} \int_{-l_{k}}^{l_{k}}\left\{K_{n k}^{33}\left(\xi, x_{n}\right)\left[\vartheta_{y_{k}}\right]^{\prime}(\xi)+K_{n k}^{34}\left(\xi, x_{n}\right)\left[\vartheta_{x_{k}}\right]^{\prime}(\xi)\right\} d \xi=m_{n}\left(x_{n}\right) \\
& \frac{D a}{4 \pi} \sum_{k=1}^{N} \int_{-l_{k}}^{l_{k}}\left\{K_{n k}^{43}\left(\xi, x_{n}\right)\left[\vartheta_{y_{k}}\right]^{\prime}(\xi)+K_{n k}^{44}\left(\xi, x_{n}\right)\left[\vartheta_{x_{k}}\right]^{\prime}(\xi)\right\} d \xi=p_{n}\left(x_{n}\right)-C_{n} \\
& x_{n} \in L_{n} \subset L_{s}, n=\overline{N_{1}+1, N}
\end{aligned}
$$

Here $m_{n}\left(x_{n}\right)=m_{n}^{0}-m_{n}^{1}\left(x_{n}\right), p_{n}\left(x_{n}\right)=p_{n}^{0}-p_{n}^{1}\left(x_{n}\right)$.

\section{Additional conditions}

$$
\left[u_{y_{n}}\right]\left( \pm l_{n}\right)=0,\left[u_{x_{n}}\right]\left( \pm l_{n}\right)=0,\left[\vartheta_{y_{n}}\right]\left( \pm l_{n}\right)=0,\left[\vartheta_{x_{n}}\right]\left( \pm l_{n}\right)=0,[w]_{n}\left( \pm l_{n}\right)=0, n=\overline{1, N}
$$

provide an one-valued solvability of singular integral equations, restoration of unknown functions by their derivatives and finding constants $C_{n}$.

Thus, the boundary problem (1)-(5) for the plate with the array of $N_{1}$ cracks and $N-N_{1}$ slits is settled to the system of $N$ singular integral equations (6) with additional conditions (7). 


\section{Results and discussion}

\subsection{Solution}

The solution to the problem (6), (7) was obtained through the asymptotic technique of small parameter and numerical method of mechanical quadratures (Savruk 1981b).

Let us use dimensionless variables $\xi=l_{k} \tau, x_{n}=l_{n} t$ and introduce new denotations:

$$
\begin{gathered}
\frac{B}{4}\left[u_{y_{n}}\right]^{\prime}\left(x_{n}\right)=f_{1 n}^{\prime}(t), \frac{B}{4}\left[u_{y_{n}}\right]^{\prime}\left(x_{n}\right)=f_{2 n}^{\prime}(t), \\
-\frac{D a}{4}\left[\vartheta_{y_{n}}\right]^{\prime}\left(x_{n}\right)=f_{3 n}^{\prime}(t),-\frac{D a}{4}\left[\vartheta_{x_{n}}\right]^{\prime}\left(x_{n}\right)=f_{4 n}^{\prime}(t) \\
l_{k} K_{n k}^{i j}\left(\xi, x_{n}\right)=K_{n k}^{i j}(\tau, t), m_{n}\left(x_{n}\right)=m_{n}(t), p_{n}\left(x_{n}\right)=p_{n}(t)
\end{gathered}
$$

Then, the equations (6) and (7) will be written as follows:

$$
\begin{aligned}
& h s_{n}(t) f_{1 n}^{\prime}(t)-\kappa f_{3 n}^{\prime}(t)=0 \\
& \frac{1}{\pi} \sum_{k=1}^{N} \int_{-1}^{1}\left\{K_{n k}^{21}(\tau, t) f_{1 k}^{\prime}(\tau)+K_{n k}^{22}(\tau, t) f_{2 k}^{\prime}(\tau)\right\} d \tau=0 \\
& \frac{h s_{n}(t)}{\pi} \sum_{k=1}^{N} \int_{-1}^{1}\left\{K_{n k}^{11}(\tau, t) f_{1 k}^{\prime}(\tau)+K_{n k}^{12}(\tau, t) f_{2 k}^{\prime}(\tau)\right\} d \tau \\
& +\frac{1}{\pi} \sum_{k=1}^{N} \int_{-1}^{1}\left\{K_{n k}^{33}(\tau, t) f_{3 k}^{\prime}(\tau)+K_{n k}^{34}(\tau, t) f_{4 k}^{\prime}(\tau)\right\} d \tau=-m_{n}(t), \\
& \frac{1}{\pi} \sum_{k=1}^{N} \int_{-1}^{1}\left\{K_{n k}^{43}(\tau, t) f_{3 k}^{\prime}(\tau)+K_{n k}^{44}(\tau, t) f_{4 k}^{\prime}(\tau)\right\} d \tau=C_{n}-p_{n}(t), \quad t \in(-1,1), n=\overline{1, N_{1}} \\
& \frac{1}{\pi} \sum_{k=1}^{N} \int_{-1}^{1}\left\{K_{n k}^{11}(\tau, t) f_{1 k}^{\prime}(\tau)+K_{n k}^{12}(\tau, t) f_{2 k}^{\prime}(\tau)\right\} d \tau=0 \\
& \frac{1}{\pi} \sum_{k=1}^{N} \int_{-1}^{1}\left\{K_{n k}^{21}(\tau, t) f_{1 k}^{\prime}(\tau)+K_{n k}^{22}(\tau, t) f_{2 k}^{\prime}(\tau)\right\} d \tau=0 \\
& \frac{1}{\pi} \sum_{k=1}^{N} \int_{-1}^{1}\left\{K_{n k}^{33}(\tau, t) f_{3 k}^{\prime}(\tau)+K_{n k}^{34}(\tau, t) f_{4 k}^{\prime}(\tau)\right\} d \tau=-m_{n}(t), \\
& \frac{1}{\pi} \sum_{k=1}^{N} \int_{-1}^{1}\left\{K_{n k}^{43}(\tau, t) f_{3 k}^{\prime}(\tau)+K_{n k}^{44}(\tau, t) f_{4 k}^{\prime}(\tau)\right\} d \tau=C_{n}-p_{n}(t), \quad t \in(-1,1), n=\overline{N_{1}+1, N} \\
& f_{\text {in }}( \pm 1)=0, \quad \int_{-1}^{1} f_{4 n}(\tau) d \tau=0, \quad i=\overline{1,4}, \quad n=\overline{1, N}
\end{aligned}
$$

We should separately discuss the multipliers $s_{n}(t)=\operatorname{sgn} f_{3 n}(t), n=\overline{1, N_{1}}$ in the first and the third equations (8). Thanks to these multipliers, the system (8) is nonlinear. 
From the definition of function $f_{3 n}(t)$, it follows that $s_{n}(t)=-\operatorname{sgn}\left[\vartheta_{y_{n}}\right]\left(x_{n}\right)$, and the line of the crack edges' contact belongs to the front surface $z=s_{n} h$. According to the initial assumption, the contact conditions (2) are followed along the whole length of each crack, and $m_{n}(t)$ have a constant sign on each $L_{n}, n=\overline{1, N_{1}}$. Then, we should hope that $s_{n}(t)$ is a constant, different for each crack: $s_{n}(t)=s_{n}, t \in(-1,1), n=\overline{1, N_{1}}$, where $s_{n}=+1$ or $s_{n}=-1$.

Thus, using the classical Kirchhoff hypothesis about the direct normal, we took down the three-dimensional problem of the elasticity with an unknown two-dimensional contact area to a two-dimensional problem of the theory of plates with unknown contact lines in front surfaces. An additional assumption about the loads of constant sign narrowed down the search for these contact lines to finding $N_{1}$ of the unknown signs $s_{n}= \pm 1$. Technically, at this stage the iterative procedure is necessary with the control over the kinematic and power inequalities in boundary conditions (2).

As we know from Savruk's monograph (1981b), the kernels of equations (8) develop in the series of the kind

$$
K_{n k}^{i j}(\tau, t)=\frac{\delta_{i j} \delta_{n k}}{\tau-t}+\sum_{p=1}^{\infty} \sum_{q=0}^{p} a_{n k p q}^{i j} \tau^{q} t^{p-q} \lambda^{p+1}
$$

where $\quad \lambda=\max _{n}\left\{2 l_{n}\right\} / \min _{n \neq k}\left\{d_{n k}\right\} \quad$ is the small non-dimensional parameter, $d_{n k}=\sqrt{\left(x_{n}^{0}-x_{k}^{0}\right)^{2}+\left(y_{n}^{0}-y_{k}^{0}\right)^{2}}$ is the distance between the centers of defects, $a_{n k p q}^{i j}$ are the coefficients, dependent on the geometry of the reciprocal location of the cuts, $\delta_{i j}$ is Kronecker delta.

Analytical results for derivative of the jumps of the displacements and rotation angles of the normal were received in the form of asymptotic series by parameter $\lambda$, which is small at long distances between the defects. Thus,

$$
f_{i n}^{\prime}(t)=\sum_{p=0}^{\infty} f_{i n, p}^{\prime}(t) \lambda^{p}, \quad C_{n}=\sum_{p=0}^{\infty} C_{n, p} \lambda^{p}
$$

By substituting the formulae (10) in the relations (8), (9) and equating the components with the same degree $\lambda$, we obtained a chain of singular integral equations with the Cauchy-type kernel, which have a closed analytical solution in the functions class with the singularity $(-1 / 2)$ on the ends of the interval.

Additionally, by assuming that $m_{n}(t)=m_{n}=$ const, $p_{n}(t)=p_{n}=$ const, $n=1,2$ we found the component of the distribution of the required functions on cracks:

$$
\begin{gathered}
f_{1 n, p}^{\prime}(t)=\frac{\kappa}{h} s_{n} f_{3 n, p}^{\prime}(t) \\
f_{2 n, p}^{\prime}(t)=\frac{1}{\sqrt{1-t^{2}}} \sum_{\substack{k=1 \\
k \neq n}}^{N} \sum_{r=0}^{p-1} \sum_{q=0}^{r} H_{r-q}(t)\left(a_{n k r q}^{21} G_{1 k, p-r-1}^{q}+a_{n k r q}^{22} G_{2 k, p-r-1}^{q}\right)
\end{gathered}
$$




$$
\begin{gathered}
f_{3 n, p}^{\prime}(t)=\frac{1}{\sqrt{1-t^{2}}}\left\{\frac{-m_{n} t}{1+\kappa} \delta_{0 p}+\right. \\
\left.+\sum_{\substack{k=1 \\
k \neq n}}^{N} \sum_{r=1}^{p-1} \sum_{q=0}^{r} \frac{H_{r-q}(t)}{1+\kappa}\left(s_{n} h\left(a_{n k r q}^{11} G_{1 k, p-r-1}^{q}+a_{n k r q}^{12} G_{2 k, p-r-1}^{q}\right)+a_{n k r q}^{33} G_{3 k, p-r-1}^{q}+a_{n k r q}^{34} G_{4 k, p-r-1}^{q}\right)\right\} \\
f_{4 n, p}^{\prime}(t)=\frac{1}{\sqrt{1-t^{2}}}\left\{\left(C_{n, p}-p_{n} \delta_{0 p}\right) t+\right. \\
\left.+\sum_{\substack{k=1 \\
k \neq n}}^{N} \sum_{\substack{r=1 \\
\sum_{q=0}-1}}^{r} H_{r-q}(t)\left(a_{n k r q}^{43} G_{3 k, p-r-1}^{q}+a_{n k r q}^{44} G_{4 k, p-r-1}^{q}\right)\right\}, n=\overline{1, N_{1}}
\end{gathered}
$$

on slits:

$$
\begin{aligned}
& f_{1 n, p}^{\prime}(t)=\frac{1}{\sqrt{1-t^{2}}} \sum_{\substack{k=1 \\
k \neq n}}^{N} \sum_{r=0}^{p-1} \sum_{q=0}^{r} H_{r-q}(t)\left(a_{n k r q}^{11} G_{1 k, p-r-1}^{q}+a_{n k r q}^{12} G_{2 k, p-r-1}^{q}\right) \\
& f_{2 n, p}^{\prime}(t)=\frac{1}{\sqrt{1-t^{2}}} \sum_{\substack{k=1 \\
k \neq n}}^{N} \sum_{r=0}^{p-1} \sum_{q=0}^{r} H_{r-q}(t)\left(a_{n k r q}^{21} G_{1 k, p-r-1}^{q}+a_{n k r q}^{22} G_{2 k, p-r-1}^{q}\right)
\end{aligned}
$$

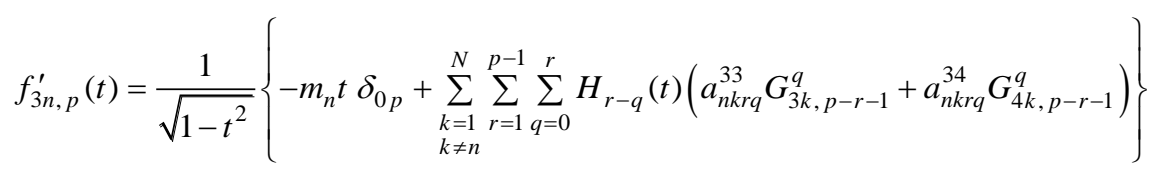

$$
\begin{aligned}
& f_{4 n, p}^{\prime}(t)=\frac{1}{\sqrt{1-t^{2}}}\left\{\left(C_{n, p}-p_{n} \delta_{0 p}\right) t+\right. \\
& \left.+\sum_{\substack{k=1 \\
k \neq n}}^{N} \sum_{r=1}^{p-1} \sum_{q=0}^{r} H_{r-q}(t)\left(a_{n k r q}^{43} G_{3 k, p-r-1}^{q}+a_{n k r q}^{44} G_{4 k, p-r-1}^{q}\right)\right\}, n=\overline{N_{1}+1, N}
\end{aligned}
$$

and also the components of the constants on all defects:

$$
C_{n, p}=p_{n} \delta_{0 p}-\frac{2}{\pi} \sum_{\substack{k=1 \\ k \neq n}}^{N} \sum_{r=1}^{p-1} \sum_{q=0}^{r}\left\{\left(a_{n k r q}^{43} G_{3 k, p-r-1}^{q}+a_{n k r q}^{44} G_{4 k, p-r-1}^{q}\right) \int_{-1}^{1} \frac{t H_{r-q}(t)}{\sqrt{1-t^{2}}} d t\right\}, n=\overline{1, N}
$$

Here $\kappa=3(1+v) /(3+v)$,

$$
\begin{gathered}
G_{i k, p}^{\alpha}=\frac{1}{\pi} \int_{-1}^{1} \tau^{\alpha} f_{i k, p}^{\prime}(\tau) d \tau, \quad H_{i}(t)=\frac{1}{\pi} \int_{-1}^{1} \frac{\tau^{i} \sqrt{1-\tau^{2}}}{\tau-t} d \tau, \quad H_{0}(t)=-t, \quad H_{2 m}(t)=t H_{2 m-1}(t), \\
H_{2 m-1}(t)=-t^{2 m}+\sum_{v=0}^{m-1} \frac{(2 m-2 v-3) ! !}{(2 m-2 v) ! !} t^{2 v}, \quad(m=1,2, \ldots ; \quad(-1) ! !=1)
\end{gathered}
$$

Thus, by integrating along $t$ derivatives in expressions (11) and (12) under the conditions (9), we determined the functions themselves $f_{i n, p}(t)$. The set $s_{n}$ may be found by the naught members of the functions' distributions $f_{3 n}(t)$, namely: 


$$
s_{n}=\operatorname{sgn} f_{3 n}(t)=\operatorname{sgn}\left(f_{3 n, 0}(t)+O\left(\lambda^{2}\right)\right)=\operatorname{sgn}\left(\sqrt{1-t^{2}}\left(m_{n}+O\left(\lambda^{2}\right)\right)\right)=\operatorname{sgn} m_{n}, \quad n=\overline{1, N_{1}}
$$

If $m_{n}=0$ on a crack or it is very insignificant compared to the load on other defects, then $s_{n}$ is determined by higher approximations.

For the factors of intensity of forces and moments, we have the expressions:

$$
K_{i n}^{ \pm}=\mp \sqrt{l_{n}} \lim _{t \rightarrow \pm 1} \sqrt{1-t^{2}} f_{i n}^{\prime}(t)=\sqrt{l_{n}} \lim _{t \rightarrow \pm 1} f_{i n}(t) / \sqrt{1-t^{2}}, i=\overline{1,4}, \quad n=\overline{1, N}
$$

We also obtained the analytical expression of contact forces on the crack lines:

$$
\begin{aligned}
& N_{y_{n}}(t, 0)=-\frac{\kappa s_{n} m_{n}}{h(1+\kappa)}+\frac{1}{1+\kappa} \sum_{p=1}^{\infty} \lambda^{p} \times \\
& \times \sum_{\substack{k=1 \\
k \neq n}}^{N} \sum_{r=0}^{p-1} \sum_{q=0}^{r} t^{r-q}\left\{G_{1 k, p-r-1}^{q} a_{n k r q}^{11}+G_{2 k, p-r-1}^{q} a_{n k r q}^{12}-\frac{\kappa s_{n}}{h}\left(G_{3 k, p-r-1}^{q} a_{n k r q}^{33}+G_{4 k, p-r-1}^{q} a_{n k r q}^{34}\right)\right\}, \quad n=\overline{1, N_{1}}
\end{aligned}
$$

\subsection{The mutual influence of two parallel defects (crack and slit)}

Let the infinite elastic plate is weakened by a dyad of two through parallel rectilinear defects the crack and the slit $\left(N=2, N_{1}=1\right)$, each $2 l$ long, with distance $d$ between the centers (Fig. 2).

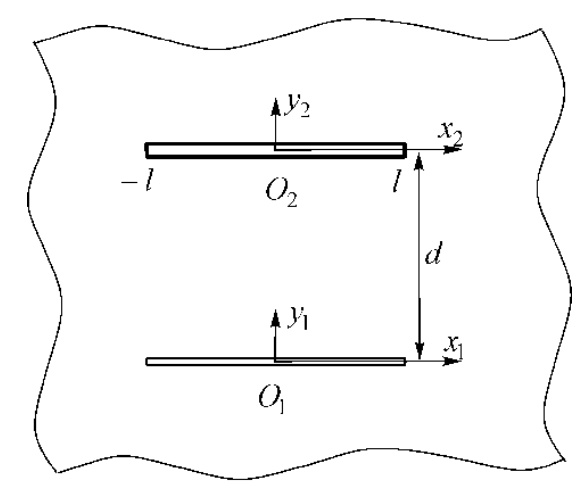

Fig. 2. Parallel crack and slit in plate under uniform bending

We considered that the plate is free from bending load on infinity $\left(m_{y}^{\infty}=m_{x}^{\infty}=m_{x y}^{\infty}=0\right)$ and under the influence of a uniform bending on each defect with the bending moment ( $m_{n}^{1}\left(x_{n}\right)=m_{n}=$ const, $\left.p_{n}^{1}\left(x_{n}\right)=0, n=1,2\right)$. Then $m_{n}(t)=m_{n}, p_{n}(t)=0, n=1,2$. For this case we obtained and investigated analytical and numeric solutions to the problem, depending on the dimensionless parameter $\lambda=2 l / d$, which characterizes mutual location of the defects. 
Namely, the coefficients of the intensity of forces and moments were calculated by the asymptotic formulae at crack:

$$
\begin{gathered}
K_{11}^{ \pm}=\frac{\sqrt{l} \kappa s_{1}}{h(1+\kappa)}\left(m_{1}-\frac{1}{8} \lambda^{2}\left(1-2 \kappa_{0}\right) m_{2}+\lambda^{4}\left(m_{1} \frac{9 \kappa+\left(1-2 \kappa_{0}\right)^{2}}{64(1+\kappa)}+m_{2} \frac{9}{128}\left(1-4 \kappa_{0}\right)\right)+O\left(\lambda^{6}\right)\right) \\
K_{21}^{ \pm}= \pm \frac{9}{128} \frac{\sqrt{l} \kappa s_{1} m_{1}}{h(1+\kappa)} \lambda^{5}+O\left(\lambda^{7}\right) \\
K_{11}^{ \pm}=\frac{\sqrt{l} \kappa s_{1}}{h(1+\kappa)}\left(m_{1}-\frac{1}{8} \lambda^{2}\left(1-2 \kappa_{0}\right) m_{2}+\lambda^{4}\left(m_{1} \frac{9 \kappa+\left(1-2 \kappa_{0}\right)^{2}}{64(1+\kappa)}+m_{2} \frac{9}{128}\left(1-4 \kappa_{0}\right)\right)+O\left(\lambda^{6}\right)\right) \\
K_{31}^{ \pm}=\frac{\sqrt{l}}{1+\kappa}\left(m_{1}-\frac{1}{8} \lambda^{2}\left(1-2 \kappa_{0}\right) m_{2}+\lambda^{4}\left(m_{1} \frac{9 \kappa+\left(1-2 \kappa_{0}\right)^{2}}{64(1+\kappa)}+m_{2} \frac{9}{128}\left(1-4 \kappa_{0}\right)\right)+O\left(\lambda^{6}\right)\right) \\
K_{41}^{ \pm}= \pm \sqrt{l} \kappa_{0}\left(\frac{3}{8} \lambda^{3} m_{2}-\lambda^{5}\left(m_{1} \frac{m_{1}}{16} \frac{\left(1-2 \kappa_{0}\right)}{1+\kappa}+m_{2} \frac{15}{64}\right)+O\left(\lambda^{7}\right)\right)
\end{gathered}
$$

and at slot:

$$
\begin{gathered}
K_{12}^{ \pm}=-\frac{\sqrt{l} \kappa s_{1}}{h(1+\kappa)}\left(\frac{3}{8} \lambda^{2} m_{1}-\lambda^{4}\left(m_{2} \frac{3}{64}\left(1-2 \kappa_{0}\right)+m_{1} \frac{45}{128}\right)+O\left(\lambda^{6}\right)\right) \\
K_{22}^{ \pm}= \pm \frac{\sqrt{l} \kappa s_{1}}{h(1+\kappa)}\left(\frac{3}{16} \lambda^{3} m_{1}-\lambda^{5}\left(m_{2} \frac{3}{128}\left(1-2 \kappa_{0}\right)+m_{1} \frac{15}{64}\right)+O\left(\lambda^{7}\right)\right) \\
K_{32}^{ \pm}=\sqrt{l}\left(m_{2}-\frac{1}{8} \lambda^{2} \frac{1-2 \kappa_{0}}{1+\kappa} m_{1}+\lambda^{4}\left(m_{2} \frac{\left(1-2 \kappa_{0}\right)^{2}}{64(1+\kappa)}+m_{1} \frac{9\left(1-4 \kappa_{0}\right)}{128(1+\kappa)}\right)+O\left(\lambda^{6}\right)\right) \\
K_{42}^{ \pm}=\mp \frac{\sqrt{l} \kappa_{0}}{1+\kappa}\left(\frac{3}{16} \lambda^{3} m_{1}-\lambda^{5}\left(m_{2} \frac{3}{128}\left(1-2 \kappa_{0}\right)+m_{1} \frac{15}{64}\right)+O\left(\lambda^{7}\right)\right)
\end{gathered}
$$

Here and hereafter $\kappa_{0}=(1-v) /(3+v)$.

Consequently, the expression for the contact reaction is:

$$
\begin{aligned}
& N_{y_{1}}(t, 0)=-\frac{\kappa s_{1}}{h(1+\kappa)} \times \\
& \times\left(m_{1}-\frac{1}{8} \lambda^{2}\left(1-2 \kappa_{0}\right) m_{2}-\lambda^{4}\left(m_{1} \frac{9-\left(1-2 \kappa_{0}\right)^{2}}{64(1+\kappa)}-m_{2} \frac{3\left(1-4 \kappa_{0}\right)}{32}\left(t^{2}+\frac{1}{4}\right)\right)+O\left(\lambda^{6}\right)\right)
\end{aligned}
$$

The sign of the function $f_{31}(t)$ overall was determined by the two members of the distribution: 


$$
s_{1}=\operatorname{sgn} f_{31}(t)=\operatorname{sgn}\left(K_{31}^{ \pm}\right)=\operatorname{sgn}\left(m_{1}-\frac{1}{8} \lambda^{2}\left(1-2 \kappa_{0}\right) m_{2}+O\left(\lambda^{4}\right)\right)
$$

If such $s_{1}$ is chosen, all contact inequalities on the crack are performed asymptotically, namely: $f_{1}(t)>0 \Leftrightarrow K_{11}>0$ and $N_{y_{1}}(t, 0) \leq 0$.

\subsection{Discussion}

The numerical analysis of the problem was done in the range of $\lambda \in[0,5]$, assuming $v=0.3$.

We considered four cases of loads on the defects' edges: 1) the crack and the slit are loaded by moments with identical signs: $m_{1}=m, m_{2}=m$;

2) the crack and the slit are loaded by moments with different signs: $m_{1}=m, m_{2}=-m$; 3 ) only the crack is loaded with a bending moment: $m_{1}=m, m_{2}=0$; 4) only the slit is loaded with a bending moment: $m_{1}=0, m_{2}=m$;

The analysis has shown that the solution to the non-linear problem is non-contradictory. In the first three cases, $s_{1}$ is defined by the sign of the load on the crack: $s_{1}=\operatorname{sgn} m_{1}=\operatorname{sgn} m$, and in the fourth case it is opposite to the sign of the load on the slit: $s_{1}=-\operatorname{sgn} m_{2}=-\operatorname{sgn} m$.

The results of the calculations, dependent on the reciprocal situation of the defects, are given in Figs. 3-6. For the coefficients of intensity of forces and moments, we have adopted the normalizing $\quad K_{1 n}=h K_{1 n}^{ \pm} /(|m| \sqrt{l}), \quad K_{2 n}= \pm h K_{2 n}^{ \pm} /(|m| \sqrt{l}), \quad K_{3 n}=K_{3 n}^{ \pm} /(m \sqrt{l})$, $K_{4 n}= \pm h K_{4 n}^{ \pm} /(m \sqrt{l}), \quad n=1,2, \quad$ and for the non-dimensional contact reaction $N_{y}=h N_{y_{1}}(t, 0) /|m|$. Solid lines on the graphs are built by the numerical data, and the dashed lines - by the asymptotic expressions (13), (14).

In case of the impact of normal bending moments on the defects, the intensity coefficients $K_{1 n}, K_{3 n}$ are dominant, which are responsible for the exposure of the defects (mode I). Here, the intensity coefficients $K_{2 n}, K_{4 n}$ (mode II) are insignificant and for this reason we will not analyze them.

In the first three instances (Figs. 3-5), when the crack is loaded, the coefficient of the intensity of the moments $K_{31}$ decreases and a considerable coefficient of the intensity of forces $K_{11}$ appears, caused by the edge contact. At the same time, a significant coefficient of the intensity of the moments $K_{32}$ is preserved on the loaded crack (cases 1), 2), 4), Figs. 3, 4) and an insignificant coefficient of the intensity of forces $K_{12}$ appears, induced by the closure of the adjacent crack. Additionally, for all four cases of the loading we observe $K_{12}<0$, which is evidence of the negative jump of the normal displacement in the median surface on the slit. In the fourth case (Fig. 6), all intensity coefficients are insignificant on the unloaded crack.

During the approximation of the defects, which is interpreted as the increase of the parameter $\lambda$, the dominant coefficients of the intensity of forces $K_{1 n}$ and moments $K_{3 n}$ decrease in the case of loading with identical sign (Fig. 3). At the same time, the increase in the parameter $\lambda$ leads to the increase of the coefficients $K_{1 n}, K_{3 n}$ in all the other cases of loading (Figs. 4-6). 

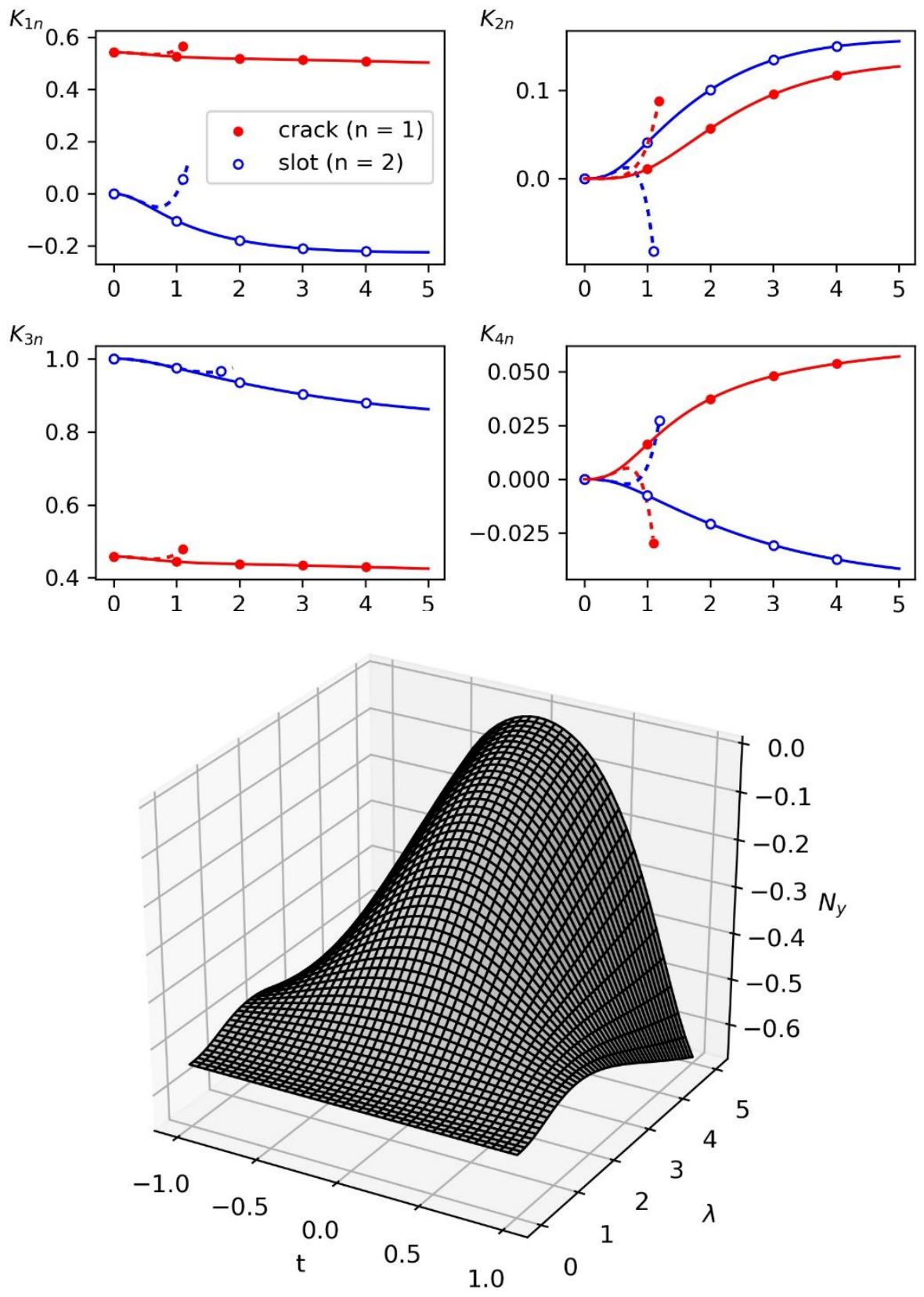

Fig. 3. The intensity factors of forces $\left(K_{1 n}, K_{2 n}\right)$ and moments $\left(K_{3 n}, K_{4 n}\right)$ and distribution of contact reaction $N_{y}$ in the case of loadings of identical sign: $m_{1}=m, m_{2}=m$

(Shatskyi and Dalyak (2018)) 

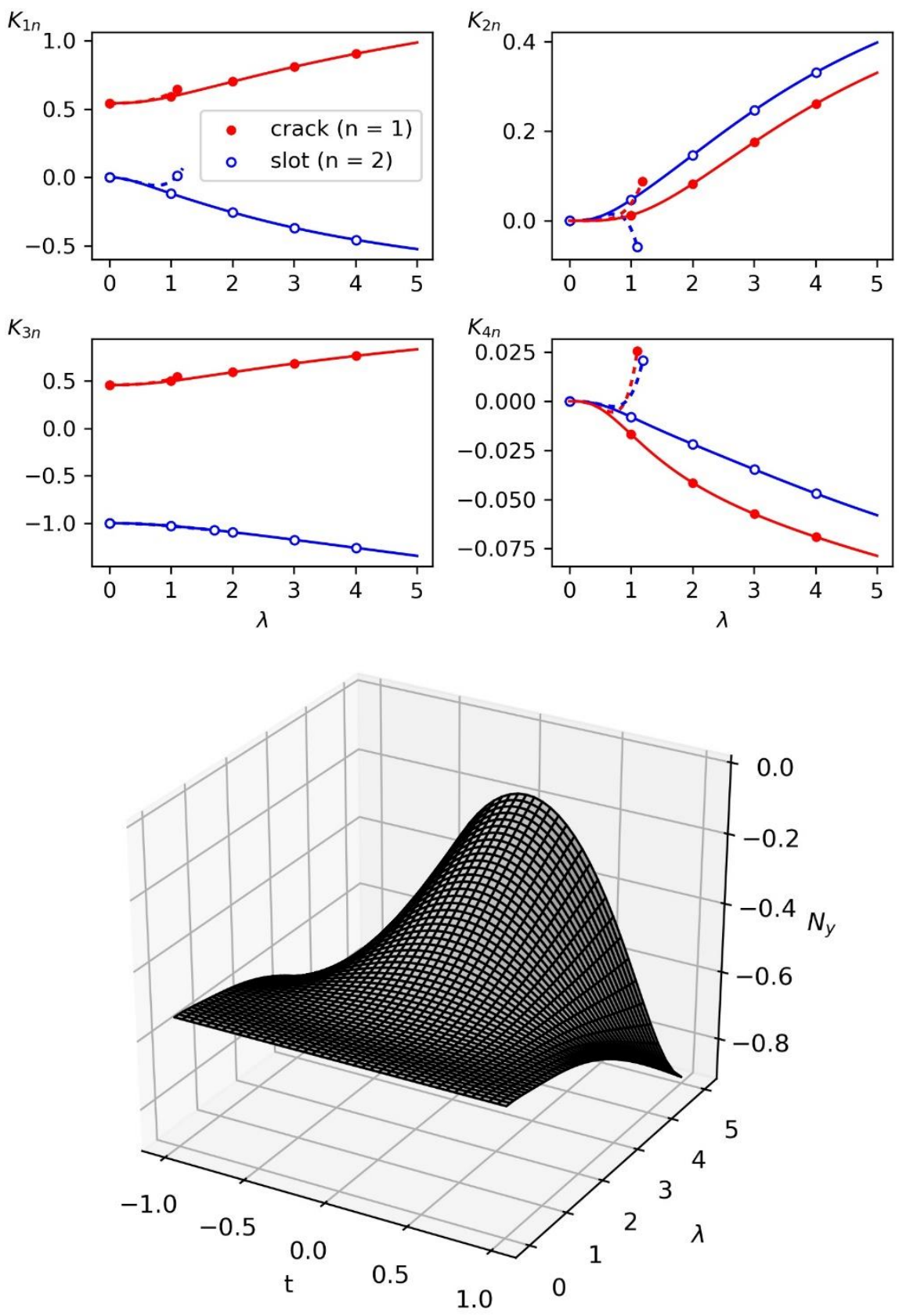

Fig. 4. The intensity factors of forces $\left(K_{1 n}, K_{2 n}\right)$ and moments $\left(K_{3 n}, K_{4 n}\right)$ and distribution of contact reaction $N_{y}$ in the case loadings of different sign: $m_{1}=m, m_{2}=-m$ 

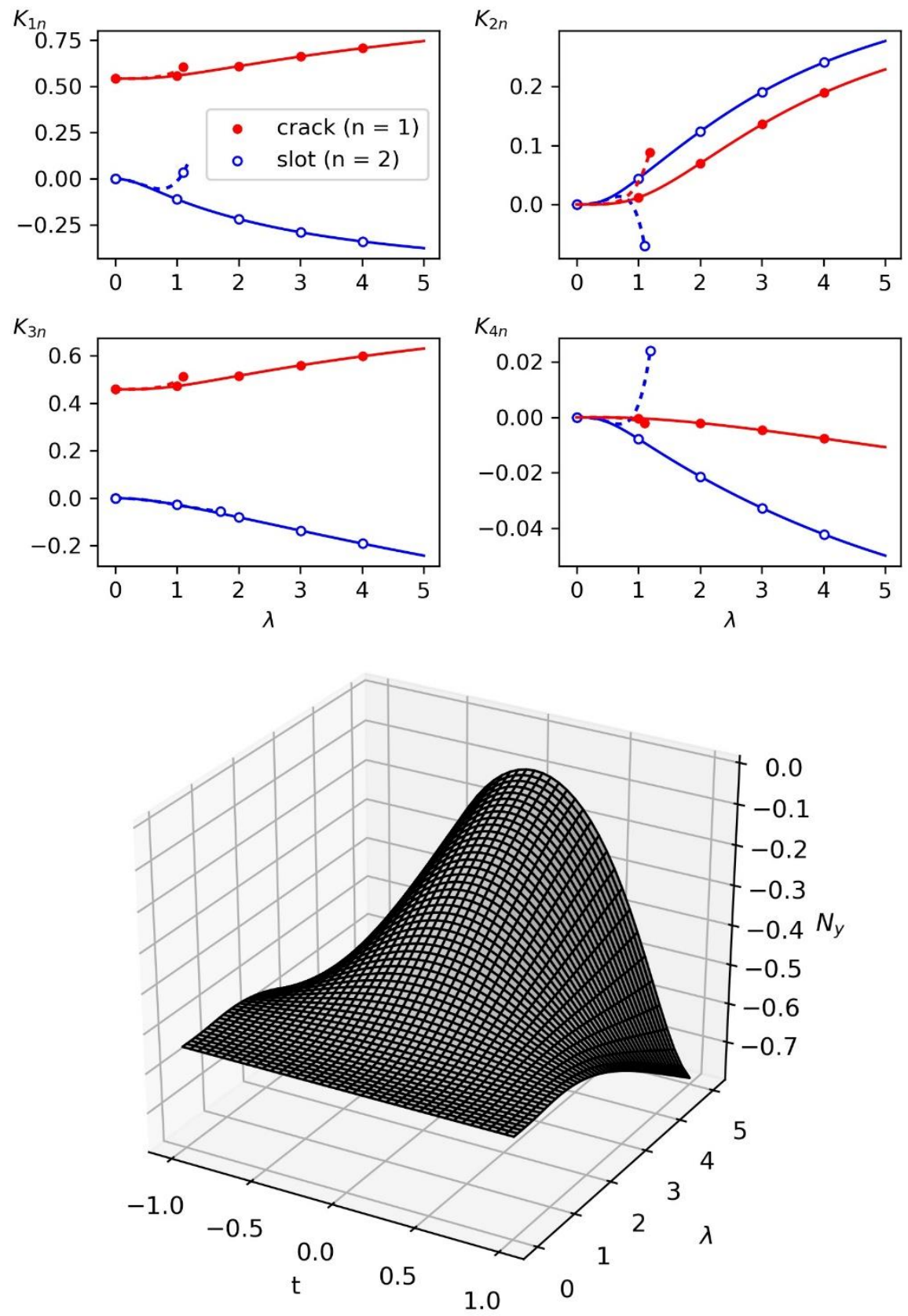

Fig. 5. The intensity factors of forces $\left(K_{1 n}, K_{2 n}\right)$ and moments $\left(K_{3 n}, K_{4 n}\right)$ and distribution of contact reaction $N_{y}$ in the case of loading of the crack edges: $m_{1}=m, m_{2}=0$ 

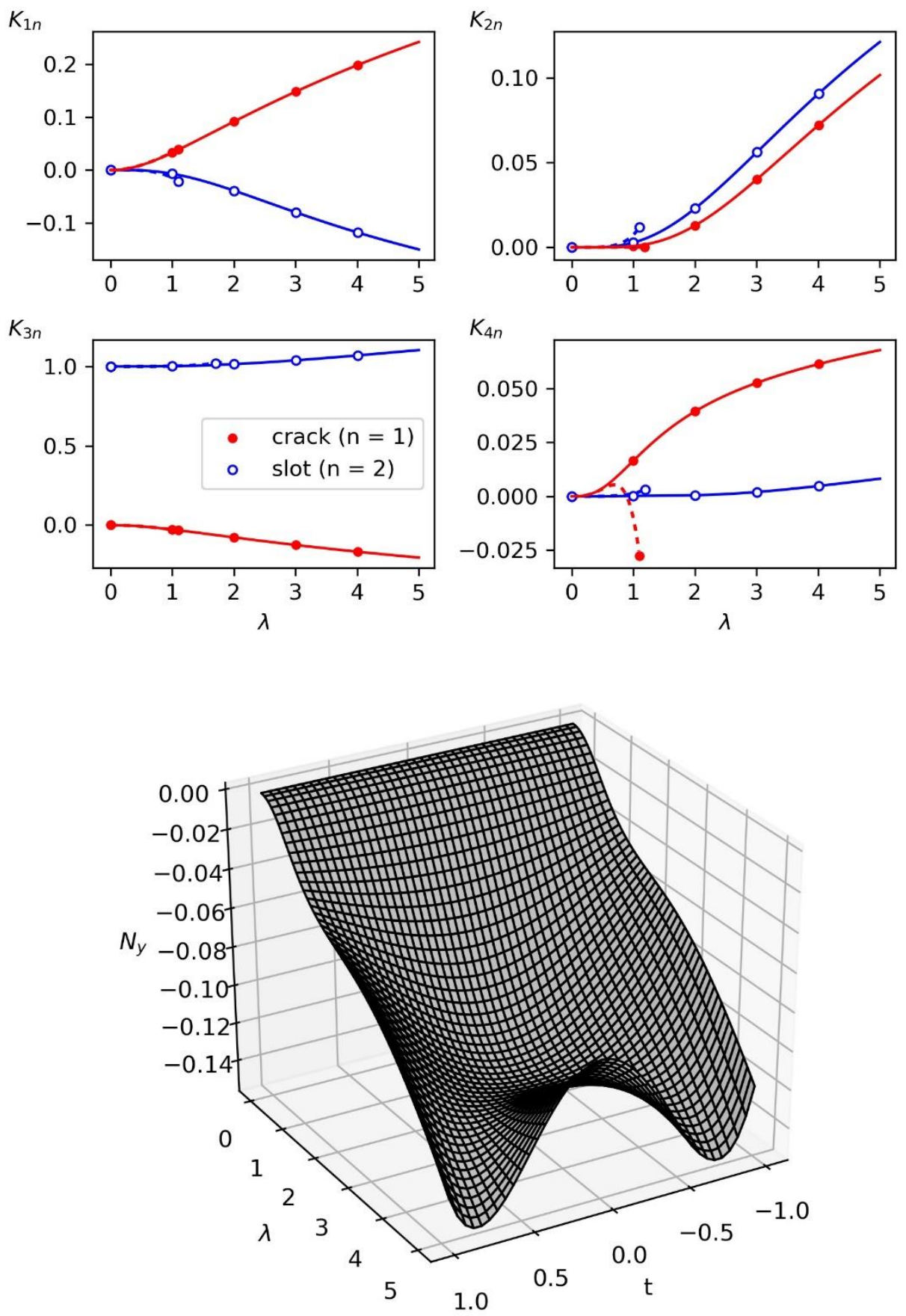

Fig. 6. The intensity factors of forces $\left(K_{1 n}, K_{2 n}\right)$ and moments $\left(K_{3 n}, K_{4 n}\right)$ and distribution of contact reaction $N_{y}$ in the case of loading of the slit edges: $m_{1}=0, m_{2}=m$ 
The regularities of the distribution of the contact reaction on the crack edges are as follows. If $\lambda$ increases, then the interaction of the edges near the middle of the crack abates in cases 1) and 3) (Figs. 3, 5) and non-monotonically depends on $\lambda$ in the case of loading with different signs (case 2), Fig. 4). Near the ends of the crack, the case is the opposite: $N_{y}$ changes nonmonotonically in cases 1), 3) and monotonically increases in case 2). The phenomenon of the reduction in the module of contact effort near the middle of the crack, located in proximity to the slit, is similar to the effect of interaction of the crack with the free edge of the semi-infinite plate (Dalyak et al. (2003)) or with a circular hole free from stress (Slobodyan (2005)).

In the fourth case of the unloaded crack, $N_{y}$ is naturally absent at $\lambda=0$, thus, the contact interaction intensifies at the approximation of the defects (Fig. 6).

Additionally, it should be noted that the presence of the cracks increases the stress field around the crack compared with the case of the interaction of two parallel cracks, studied by Shats'kyi and Dalyak (2000).

For small values of $\lambda$, the same behavior of intensity factors and contact reaction is qualitatively represented also by asymptotic formulae (13)-(15). If we accept the numerical results as accurate, then the relative error of the calculation of significant coefficients of intensity by the formulae (13), (14) at $\lambda<1($ read at $d>2 l)$ does not exceed $4 \%$.

\section{Conclusions}

We have suggested a calculation scheme, which allows research of the effects of interaction of closable (contact) cracks and narrow slits within the assumes of the classic theory of plates. By the method of singular integral equations, we have constructed an asymptotic solution to the problem of a series of randomly situated rectilinear defects.

On the bases of the asymptotic and numerical solutions, obtained for the pair of interacting parallel defects of varying nature at four types of bending stress, it has been determined that a crack closure decreases the bending moments intensity in the neighborhoods of the tips of the crack, and it also produces nonzero value of coefficients of intensity of membrane forces in the peaks of both defects.

The accuracy of the obtained asymptotic formulae is acceptable if the distance between the defects exceeds their length.

\section{References}

Berezhnitskii LT, Delyavskii MV, Panasyuk VV (1979). Bending of thin plates with defects such as cracks, Naukova dumka, Kiev.

Bozhidarnik VV, Opanasovich VK, Gerasimchuk PV (2006). Bilateral bending of a plate with nonsymmetric through-thickness arc crack with allowance for the contact of its edges, Strength of Materials, 38(5), 548-553.

Dalyak TM (2004). Bending of a plate containing a periodic system of shifted parallel cracks whose lips are in contact, Materials Science, 40(1), 139-143.

Dalyak T (2019). Investigation of the interaction of two parallel shifted cracks in plate bending adjusted for their closure, Theoretical and Applied Mechanics, 46, 147-155

Dalyak T, Perepichka V, Shats'kyi I (2003). Closure of an arbitrarily oriented crack at bending of a half-infinite plate, Mashynoznavstvo, 9, 11-15. 
Dovbnya KM, Hryhorchuk YuV (2016). Stressed state of shell of double curvature with two collinear cracks under bending, Journal of Mathematical Science, 212(1), 98-105.

Dovbnya KM, Shevtsova NA (2014). Two collinear cracks with contacting lips in an orthotropic shell of any curvature under the conditions of bending, Materials Science, 49(6), 743-748.

Heming FS (1980). Sixth order analysis of crack closure in bending of an elastic plate, International Journal of Fracture, 16(4), 289-304.

Isida M (1977). Bending of plate containing arbitrary array of cracks, Transactions of the Japan Society of Mechanical Engineers, 43(367), 825-837.

Jones DP, Swedlow JL (1975). The influence of crack closure and elasto-plastic flow on the bending of a cracked plate, International Journal of Fracture, 11(6), 897-914.

Khludnev AM (1995). On contact problem for a plate having a crack, Control and Cybernetics, 24(3), 349-361.

Khludnev AM, Kovtunenko VA (2000). Analysis of cracks in solids, WIT-Press, Southampton; Boston.

Lazarev NP (2011). An equilibrium problem for a Timoshenko plate with a through crack, Sib. Zh. Ind. Mat., 14(4), 32-43.

Liu R, Wang CH, Bathgate RG (1999). Crack closure in spherical shells, International Journal of Fracture, 99(4), 307-323.

Murakami Y (1987). Stress Intensity Factors Handbook, 2, Pergamon Press.

Opanasovych V, Seliverstov R (2006). The influence of closure of two collinear cracks on the stress state of transversal-isotropic plate under pure bending, Visnyk of the Lviv University, Series Mechanics and Mathematics, 65, 152-157.

Opanasovych VK, Slobodyan MS (2018). Bending of an Isotropic plate with two identical coaxial through cracks depending on the width of the contact zone of their faces and in the presence of plastic zones near their tips, Journal of Mathematical Sciences, 229(3), 280-291.

Opanasovych VK, Stashchuk MH, Dorosh MI (2008). Bending of a plate containing a periodic system of collinear cracks with regard for the contact of crack lips, Materials Science, 44(2), 201-210.

Perepichka VV (1998). Bending of a semi-infinite cantilevered plate weakened by a cut with contacting edges, Journal of Mathematical Sciences, 90(2), 1978-1981.

Perepichka VV, Shats'kyi IP (2002). Bending of a semi-infinite plate with a periodic system of cuts considering the contact of their edges, Journal of Mathematical Sciences, 109(1), 12901294.

Savruk MP (1981a). Bending of thin elastic plates weakened by curved cracks, Materials Science, 16, 369-375.

Savruk MP (1981b). Two-dimensional problems of elasticity for cracked bodies, Naukova dumka, Kiev.

Shats'kyi IP (2015). Limiting equilibrium of a plate with partially healed crack, Materials Science, 51(3), 322-330.

Shats'kyi I, Dalyak T (2000). Mutual influence of parallel cracks, the banks of which contact in plate bending, Mashynoznavstvo, 1, 27-30.

Shats'kyi IP, Dalyak TM (2002). Closure of cracks merged with slots in bent plates, Materials Science, 38(1), 24-33.

Shats'kyi IP, Makoviichuk MV (2005). Contact interaction of crack lips in shallow shells in bending with tension. Materials Science, 40(4), 486-494.

Shats'kyi IP, Makoviichuk MV (2009). Analysis of the limiting state of cylindrical shells with cracks with regard for the contact of crack lips, Strength of Materials, 41(5), 560-565.

Shats'kyi IP, Perepichka VV (2004). Limiting state of a semi-infinite plate with edge crack in bending with tension, Materials Science, 40(2), 240-246.

Shatskii IP (1989). Contact of the edges of the slit in the plate in combined tension and bending, Materials Science, 25(2), 160-165. 
Shatskii IP (1990). Interaction of collinear sections with contacting edges in a bent plate, Materials Science, 26(3), 311-316.

Shatskii IP (1991). Bending of a plate containing a periodic system of parallel slits with contacting edges, Soviet Applied Mechanics, 27(12), 1186-1190.

Shatskii IP (2001). Model for contact of crack boundaries in a bending plate, Journal of Mathematical Sciences, 103(3), 357-362.

Shatskii IP, Makoviichuk NV (2011). Effect of closure of collinear cracks on the stress-strain state and the limiting equilibrium of bent shallow shells, Journal of Applied Mechanics and Technical Physics, 52(3), 464-470.

Shatsky IP (1988). Bending of the plate weakened by the crack with contacting edges, Dopovidi Akademii Nauk Ukrainskoi RSR. Ser. A - Fiziko-matematichni ta technichni nauki, 7, 4951.

Shatsky IP (1998). A cracks closure in combined tension and bending of plates, Fracture from detects, Proc. 12 th Bien. Conf. of Fract, ECF-12, Sheffield, Ed. M.W.Brown, 2, 733-738.

Shatskyi IP, Dalyak TM (2015). Interaction of crack and collinear slot in plate bending, Bulletin of Zaporizhzhia National University, Physical \& Mathematical Sciences, 1, 211-218.

Shatskyi I, Makoviichuk M, Perepichka V, Dalyak T (2017). Effect of cracks closure in plates and shells under combined tension and bending, Engineering mechanics, 23rd Int. Conf., Book of full texts, Ed., V. Fuis, 866-869.

Shatskyi I, Dalyak T (2018). Interaction of contact cracks and narrow slits in plate bending, Procedia Structural Integrity, 13, 1476-1481.

Slobodyan M (2005). Two-sided bending of a plate with a circular hole and a crack, placed parallel to the diameter, taking into account the contact of its edges, Mashynoznavstvo, 5, $41-47$.

Syasky A, Muzychuk K (2012). Bending of piecewise homogeneous plate with one curvilinear cut under contacting of its edges, Visnyk TNTU, 66(2), 7-15.

Williams ML (1961). The bending stress distribution on the base of a stationary crack, Journal of Applied Mechanics, 28(1), 78-82.

Young MJ, Sun CT (1992). Influence of crack closure on the stress intensity factor in bending plates - A classical plate solution, International Journal of Fracture, 55, 81-93.

Zehnder AT, Viz MJ (2005). Fracture mechanics of thin plates and shells under combined membrane, bending and twisting loads, Applied Mechanics Reviews, 58, 37-48. 